\title{
LOS PROBLEMAS AMBIENTALES: UN NUEVO LLAMADO A LA Vita Activa DE LA FILOSOFÍA
}

\author{
ALEXANDER MARTÍNEZ RIVILLAS ${ }^{1}$ \\ Recibido el 16 de febrero de 2012 y aprobado el 19 de \\ agosto de 2012

\section{RESUMEN} \\ Se expone de forma general el impacto que la \\ problemática ambiental ha tenido en los sistemas de las \\ ciencias naturales y humanas; y en particular, las \\ principales suscitaciones intelectuales que ha generado en \\ la filosofía occidental. A la luz de este panorama, se tratará \\ de resolver una "axiomática" para una filosofía de la \\ sostenibilidad.
}

\section{PALABRAS CLAVE:}

Filosofía ambiental, ciencias ambientales, filosofía de la sostenibilidad, ecología y complejidad.

\section{"ENVIRONMENTAL PROBLEMS: A NEW CALL TO THE PHILOSOPHY Vita Activa"}

\begin{abstract}
The impact the environmental problems have generated in natural and human sciences systems is presented in a general way, particularly the main intellectual arousal derived from Western philosophy. In the light of this outlook, an "axiomatic" approach for a philosophy of sustainability will be proposed.
\end{abstract}

\section{KEY WORDS:}

Environmental philosophy, environmental science, philosophy of sustainability, ecology and complexity.

\section{INTRODUCCIÓN}

¿Qué dicen hoy la metafísica moderna y los discursos postmodernos acerca del drama del cambio climático? ¿Qué responderían hoy los filósofos más comprometidos con la profundización de la democracia ante la vieja pregunta kantiana: ..."qué nos es permitido esperar", en un mundo en el que lo esperable no depende del uso público de la razón, ni de las mejores reformas inspiradas en el "republicanismo kantiano" -sobre todo de 
cuño rawlsiano, sino de acciones inmediatas para paliar los procesos de des-fundamentación de lo que Alfred Schmidt llamaba "el primado ontológico de la naturaleza", esto es, la fuente nutricia de toda forma ontológica y, por tanto, de toda forma "históricoontológica", a decir de Heidegger..., en fin, de esta nave de dimensiones finitas en que consiste el mundo natural? ¿Estarían dispuestos los filósofos de hoy a hacer eco de las justificaciones teleológicas de la economía neoclásica, según las cuales el calentamiento global y el agotamiento de los recursos no renovables no constituyen ningún obstáculo para el progreso de la humanidad (dado el carácter sustituto que el capital le ofrece a los recursos naturales y los avances inusitados de la tecnología, según la opinión de Aalbers, Beckerman, Solow, 1997 y Stiglitz, 1997), no obstante la bizarra naturaleza que seguramente encontrarán las futuras generaciones?

Estas serán pues las cuestiones que se tratarán de resolver, siguiendo el siguiente plan expositivo: en primer lugar, se hará una breve presentación de los antecedentes de la filosofía ambiental; en segundo lugar, se explicarán los cambios más importantes que el drama ambiental ha generado en los sistemas de las ciencias naturales y humanas; en tercer lugar, se expondrán los desafíos que conlleva para la filosofía en los órdenes político, económico, ético y epistemológico, la problemática ambiental; y finalmente se hará una propuesta de mínimos principios que podrían dar soporte a una filosofía de la sostenibilidad.

\section{ANTECEDENTES DE LA FILOSOFÍA AMBIENTAL}

Tal como han nacido distintas disciplinas filosóficas durante el siglo $X X$, la filosofía ambiental encontró sus raíces en una profunda cantera de investigaciones científicas y problemas públicos de compleja resolución. En 1866, Ernst Haeckel, acuñó el término "ecología", iniciando así una larga tradición de trabajos sobre la "interacción de sistemas vivientes con sus entornos ambientales".

Posteriormente, entre los años veinte y cuarenta del siglo XX (Volterra, 1927; Gause, 1934), se erigió la denominada edad de oro de la ecología, cuyo enfoque fue, ciertamente, el estudio determinista de las poblaciones en entornos naturales determinados. Al final de dicho período, Tansley propuso, en 1935, la expresión "ecosistema", con el propósito de integrar variables físicas ala dimensión ecológica, en una inédita aspiración de integrar las ciencias físicas con las biológicas.

Dada la popularidad que alcanzó tal término durante los años cincuenta y sesenta, se inaugura una etapa de investigaciones ecosistémicas de enfoque "holístico" que habrá de contribuir al nacimiento de las disciplinas de la "complejidad" (entre las cuales se destacan los estudios de Hubbard Brook en los años sesenta), cuyo principio de investigación más simple fue "explicar todo en función de sus partes". 
En esta etapa de enfoque holístico, también se destacan las adelantadas investigaciones de ecología social que habría de dar nacimiento al biorregionalismo de Lewis Mumford (1944 y 1961), y que posteriormente fundamentaría algunos discursos de autodesarrollo de las políticas de desarrollo regional y local (aquí debemos reconocer la fuerte relación que estas narrativas tuvieron con el socialismo del siglo XIX del geógrafo Elisée Reclus y en el anarquismo de Kropotkin (Brennan \& Lo, 2008).

Paralelamente, en este mismo período, aparecen los consabidos problemas ambientales de impactos regionales y planetarios, entre los cuales se destacan los fenómenos de lluvia ácida en Europa del Norte, los derrames de petróleo que alteraron distintos ecosistemas costeros, y los diferentes daños que a la salud humana había dejado la aplicación de agroquímicos como el DDT, Aldriny Deildrin.

Ante estos nuevos dramas ecológicos, una pléyade de científicos inició una nueva tradición de investigaciones ambientales desde enfoques ecológicos integrales, esto es, estudios multidisciplinarios que agrupaban no sólo saberes físicos y biológicos de los ecosistemas, sino también sus diversas relaciones con las comunidades humanas. Se trataba entonces de aplicar el sistema de las ciencias del hombre al drama ambiental, en cuyo escenario la filosofía cumplió una magra labor. Hablamos, desde luego, de los trabajos de Rachel Carson (1963) sobre el impacto social y ambiental de los agroquímicos, de Lynn White jr. (1967) acerca de las raíces históricas de la crisis ambiental, y de Paul Ehrlich (1968) sobre la viabilidad de la vida humana en escenarios de superpoblación, entre las investigaciones más destacadas.

Pese a lo anterior, sólo fue en la década de los setenta que eclosionaron las más variadas narrativas ambientales que habrían de influenciar el debate de la crisis ambiental hasta nuestros días, entre las cuales se encuentran las primeras reflexiones filosóficas sistemáticas de cuño ambientalista. Así pues, aparece en escena el estudio Los Límites al Crecimiento de Dennis Meadows y Jay Forrester (1972), que inaugura la corriente de "modelizaciones" matemáticas sobre el comportamiento de los recursos naturales bajo la presión del crecimiento económico; las doctrinas de derecho ambiental del norteamericano Christopher Stone (1972); la revolucionaria obra del economista y matemático GeorgescuRoegen La Ley de la Entropía y el Proceso Económico (1971), que echaría las bases de la economía ecológica y fundamentaría las principales críticas científicas de hoy a la economía neoclásica y las políticas económicas liberales, junto con los destacados trabajos sobre los vínculos entre economía y ecología de Barry Commoner, Edward Goldsmith y Howard Odum, siendo este último el gran ordenador conceptual de la doctrina ecológica.

Finalmente, las primeras expresiones filosóficas que darían lugar a un sinnúmero de trabajos por sus polémicos supuestos e inusitadas 
consecuencias éticas y políticas, esto es, el movimiento de "Ecología Profunda", nacido en los países escandinavos, y el movimiento "Ética de la Tierra", nacido en Norteamérica. El primero, cuyos fundadores fueron el filósofo noruego Arne Næss, Sigmund Kvaløy y Nils Faarlund (1973), quienes impulsaron una corriente de la filosofía ambiental cuyo principio de interpretación-acción es el "igualitarismo biosférico" (se le conoce también como "ecosofía, ecosofía T, biocentrismo", y en distintas versiones se le identifica con el "panpsiquismo o animismo"). Y el segundo movimiento, cuyo fundador fue Aldo Leopold (1949), según el cual todos los intereses individuales deben someterse al bien común de la comunidad biótica de la tierra, también constituye un referente clave que habría de suscitar la atención de la filosofía (Brennan \& Lo 2008).

Asimismo, se debe reconocer la influencia significativa que en el incipiente debate de la filosofía ambiental tuvieron los siguientes filósofos o corrientes filosóficas: el australiano Richard Routley (1973), quien libró un debate frontal contra el "antropocentrismo"; el filósofo norteamericano Holmes Rolston (1975), cuyas reflexiones asignaban un valor intrínseco a todas las especies de la naturaleza, por lo cual a los hombres les era imperativo respetarla y protegerla; el "ecofeminismo", que tiene en Sheila Collins (1974) su principal representante, arguyendo que la destrucción ecológica obedecía, principalmente, a la "cultura patriarcal que extiende el dominio de lo femenino al dominio de lo natural "por una simple asociación simbólica (Brennan \& Lo 2008); el "nuevo animismo", de más reciente fundación, fuertemente inspirado en el movimiento "Ecología Profunda", aspirando a la "restauración de las prácticas ceremoniales y rituales de los pueblos indígenas" (u otras experiencias espirituales antiguas como el Taoísmo y el Budismo) a fin de establecer de nuevo los vínculos sagrados con la naturaleza viviente, esto es, la naturaleza animada y no animada (Abram, 1996 y Mathews, 2003); y finalmente, "la teoría crítica ambiental o ecocrítica", que hunde sus raíces en la Escuela de Frankfurt, cuya máxima ambiental se reconoce en el desmonte sistemático del positivismo e instrumentalismo de la racionalidad occidental, con el objeto de instaurar los valores estéticos y morales que garanticen la inauguración de una nueva era de libertad, espontaneidad y creatividad (Vogel 1996 \&Luke, 1997); lo que en efecto compelerá al hombre al "reencantamiento" de la naturaleza mediante aquella experiencia estética renovada (Brennan\& Lo, 2008).

Así pues, se han resumido las principales investigaciones científicas y filosofías ambientales que han terciado en el debate de la crisis ecológica de nuestro tiempo. Ahora, se tratará de presentar los principales impactos que este debate ha generado en el sistema de las ciencias humanas y naturales.

\section{LAS PRINCIPALES RUPTURAS EN EL SISTEMA DE LAS CIENCIAS}

Tenemos entonces cinco disciplinas en el centro de las rupturas del 
sistema de la ciencia: la biología, especialmente la ecología; la física, en particular, la termodinámica; la economía, especialmente la economía neoclásica; la sociología, básicamente la sociología ambiental; y, por supuesto, la filosofía, particularmente la filosofía de la ciencia, la filosofía de la ética, la filosofía política, la filosofía del derecho y la filosofía de la naturaleza.

En lo que corresponde a la ecología, las investigaciones de las últimas tres décadas cuestionaron profundamente la pertinencia de modelos deterministas para estudiar las poblaciones de un sistema ecológico determinado, pues los individuos de un entorno mostraban comportamientos más aleatorios y complejos que los estudiados en el entorno de la investigación piloto (Sarkar, 2005). Los comportamientos objeto de investigación, aunque se limitaban a tasa de crecimiento, riqueza y diversidad de los entornos ecológicos, exhibieron dificultades para la determinación de patrones (modelos de crecimiento, Volterra, 1927, y modelos de crecimiento logístico, Gause, 1934), cuestionaron las definiciones clásicas de diversidad, estabilidad y riqueza de las especies, en tanto que no hay hechos inequívocos que muestren que la diversidad garantice con el tiempo la riqueza, ni que la riqueza asegure la preservación de una especie, ni que la estabilidad sea asegurada mediante la diversidad y/o la riqueza de las especies (Sarkar 2005). E igualmente, en lo que corresponde a la agroecología, aún subsiste el reto de determinar y regular en el nivel local y regional una política agroalimentaria sana, segura y autosuficiente; lo que también implica estudiar pormenorizadamente, para todas las latitudes, las condiciones de humedad, nutrientes y el ciclo biológico del suelo para distintos cultivos clave (Pratley, 1989). Hoy debemos reconocer que la ecología se enfrenta a verdaderas "cajas negras", lo que en efecto exige la refinación de sus aparatos conceptuales y la especialización de sus instrumentos de medición de cara a la formulación de recomendaciones y estrategias eficientes para definir reservas biológicas, conservar ecosistemas y asegurar la eficiencia de un sistema agroalimentario ambientalmente sostenible.

En lo que toca a la termodinámica, los avances de la economía ecológica han exigido mejores métodos de medición y cuantificación de flujos de materiales y energía tanto de recursos de uso endosomático (de consumo humano) como de uso exosomático (de artefactos manuales o mecánicos) (Martínez y Roca, 2001: pp. 22-23), con el propósito de evaluar los niveles de agotamiento y disipación de materiales que proveen energía o que suministran insumos; y asimismo, contribuir con información suficiente para el diseño de políticas ambientales de nivel local, regional y mundial para el aprovechamiento sostenible de los recursos naturales renovables y no renovables. De hecho, la física, a tono con dichas exigencias ambientales, desde ya debería recomponer sus prioridades y hacer los respectivos esfuerzos en esta materia. 
En lo relativo a la economía, los retos no son sólo de carácter formal, sino también instrumental. Después de las demoledoras críticas a la economía neoclásica de Georgescu-Roegen (1971, 1972 y 1977) y Herman Daly (1977, 1980 y 1997), entre los más destacados, la economía convencional se ha visto profundamente cuestionada desde sus axiomas mismos hasta sus fórmulas canónicas. En efecto, la función de producción no opera para economías campesinas y "subdesarrolladas", pues un factor de producción adicional no garantiza de ningún modo un incremento adicional de la producción; la función de sustitución no aplica entre los niveles alimentarios y supra-alimentarios de la economía de consumo, pues "nadie reemplaza un cantidad determinada de carne por un par de zapatos"; la "función de utilidad" no opera expeditamente en comunidades indígenas, en poblaciones con familias típicamente nucleares y en sociedades campesinas, pues el patrón de conductas del "Homo Oeconomicus" no es funcional a sus usos y costumbres. Y en general, ninguna función característica de la economía neoclásica puede registrar el hecho simple de que se agotan y disipan recursos no renovables, y que las generaciones futuras deben participar de algún modo en los cálculos de los costos y beneficios de cualquier política de uso de los recursos naturales (Carpintero 2006). Ante este panorama, la economía debe ser menos convencional y más consistente con las realidades de buena parte de la población mundial, fundar nuevas funciones explicativas de las conductas económicas del individuo solidario y las comunidades colectivistas, y afinar los mecanismos de evaluación y valuación de la economía de los recursos naturales que permitan introducir la noción de finitud de los mismos y de los límites al crecimiento en virtud de la dinámicas propias de los ciclos bio-geoquímicos de la naturaleza.

En lo que hace referencia a la sociología, los cambios de paradigmas han sido sustanciales. La sociología ambiental, mediante sus "evaluaciones ambientales participativas e integradoras" (Funtowicz \& Ravetz, 1991; Renn et. al.,1995; Jäeger et. al.,1999; Font \& Subirats, 2000; Tàbara, 2003), ha efectuado una ruptura epistemológica sobre la construcción social de conocimiento y de políticas de intervención ambiental, pues han logrado posicionar el conocimiento experto, el conocimiento cotidiano del ciudadano y la esfera política en un mismo plano de importancia, lo que de hecho ha permitido establecer puentes de comunicación e investigación transparentes con la ayuda de modelizaciones matemáticas y metodologías cualitativas (Renn et. al.,1995; Munda, 1995; Munda et. al.,1995; Tàbara, 1998 \& 2003). En efecto, bajo la influencia de Habermas, especialmente en lo tocante a los modelos de comunicación transparente, se han desarrollado aquellas transformaciones epistemológicas que, antes que construir "verdades" solipsistas y positivistas, construyen "verdades" de investigación-acción, esto es, que interpretan sucesos desde distintas y abundantes perspectivas, encuentran patrones multidimensionales en los sucesos, y prescriben acciones 
para tratar de cambiarlos o mejorarlos con cierta eficiencia local. En fin, se trata de "verdades" más congruentes con la complejidad de la realidad social, a decir de Edgar Morin (1998).

\section{REPERCUSIONES EN LAS DISCIPLINAS FILOSÓFICAS}

\section{LA FILOSOFÍA DE LA CIENCIA}

Las consecuencias para la filosofía de la ciencia han sido enormes. Los estudios deterministas de la ecología fueron fuertemente cuestionados desde los años cincuenta para dar lugar a sistemas de interpretación que podemos llamar de carácter complejo. Dichos sistemas recurrieron a modelos estocásticos para analizar el universo complejo de las reservas ecológicas, especialmente la Mínima Población Viable (MPV) (Shaffer, 1978), pero, a pesar del entusiasmo, sus investigadores reconocieron incertidumbres estructurales en el modelo desde los años ochenta (Sarkar, 2005). Por otro lado, desde los años sesenta, la cibernética empezó a encontrar serias dificultades para automatizar acciones motrices inteligentes de estímulos y respuestas múltiples. Y el meteorólogo Edward Lorenz, en un proceso intenso de construcción de modelos de predicción atmosférica, que involucraban sólo tres variables: velocidad del viento, presión del aire y temperatura, determinó que la no linealidad de sus modelos registraban una suerte de "sistema caótico de retroalimentación reiterada" que, por supuesto, era bastante congruente con el tiempo atmosférico real (Briggs \& Peat, 1999: pp.43-45). Ante estos hitos científicos, y otros de mayor o menor importancia, otra pléyade de investigadores fundaron las disciplinas del "caos" o de "la complejidad".

Tales disciplinas, sistematizadas hoy a pesar de la complejidad del tema, han expuesto tres principios básicos: la realidad es una suerte de conjugación del orden y el desorden, la racionalidad puede "encerrar" elementos ordenados de la realidad, pero no elementos caóticos, y la ciencia debe continuar explicando aquella realidad que se manifieste ordenada, pero admitiendo y reconociendo que siempre se manifestará un desorden inherente (Morin, 1998).

En este orden de ideas, la filosofía ha visto con perplejidad cómo los sistemas inductivos y deductivos de las ciencias han ingresado a un verdadero pandemonio de especulaciones, pero también a una fase de autoevaluación rigurosa sobre las implicaciones de un hecho irrevocable, esto es, que la realidad es compleja. En efecto, las ciencias de la naturaleza están recurriendo a todo un arsenal de modelos no lineales para explicar fenómenos cada vez más esquivos y "caprichosos" frente a sus narrativas lógicas y matemáticas. Lo que a su vez no sólo ha generado un fuerte reconocimiento de la multidisciplinariedad de las investigaciones científicas, sino también una especie de discursividad sobre las condiciones de consensualidad de la verdad en la comunidad científica. Esto es, a falta de certezas frente a fenómenos 
complejos, que sea una suerte de elección social la que decida sobre la verdad de dichos fenómenos. Ciencia, ética y política se ven cara a cara a fuerza de complejidades fenoménicas.

"Para la muestra un botón". En la Cumbre de la Tierra en Río (1992), se instauró el principio de precaución para prevenir aquellas acciones humanas que pudieran ocasionar daños ambientales serios o irreversibles, aún si no se tuviera certeza científica suficiente sobre el peligro de dichas acciones.

En efecto, se trata de un típico problema de vínculo entre causa y efecto. Pero, ¿Cómo determinar la causa ante el evento de que un pesticida aplicado en cualquier parte del mundo haya ocasionado malformaciones genéticas en Turquía? ¿Cómo determinar el responsable del incremento de la temperatura de la tierra si no podemos argüir con plena certeza que los gases de efecto invernadero son su causa directa? ¿Cómo determinar si las fibras de asbesto contenidas en productos de libre circulación en Colombia causan cáncer de pulmón, si no disponemos de pruebas inequívocas de que haya sido el agente causante? En otras palabras, ¿cómo determinamos la "suficiente certeza" para poder dirimir el espacio de aplicación del principio de precaución? (sobre el problema del vínculo causa-efecto en esta materia véase Baker, 2007). Ciertamente, ante sucesos complejos no existen monocausalismos. Pero, a pesar de los hechos, ¿cuál será la nueva epistemología que a lo menos pueda contribuir a resolver el problema del principio de precaución? Este es uno de los más grandes desafíos que puede tener la filosofía de la ciencia para contribuir a la sostenibilidad del planeta.

\section{LA FILOSOFÍA DE LA ÉTICA}

En lo relativo a la filosofía de la ética, la fundamentación de la ética ambiental ha puesto de presente la constitución antropocéntrica del mundo occidental no sólo en lo que hace referencia al descentramiento del yo moderno en la sociedad (pluralidad ética), sino también en lo relativo al descentramiento del yo moderno en la naturaleza (moralidad hacia la naturaleza). De este modo, existe una paradoja difícil de resolver en ética ambiental, esto es, ¿para superar la crisis ambiental, el mundo de la especie humana, de los entes vivientes no humanos y de los seres inanimados, deberían gozar de los mismos derechos y respetos morales, no obstante la pluralidad cultural y religiosa que contradice dicho igualitarismo biosférico?, o dicho de otra manera, ¿dado que el drama ambiental requiere, ante todo, de cambios en las conductas cotidianas de los ciudadanos (la modernización ecológica y el dumping ambiental tienen por supuesto fundamental tal cambio, Lenschow, 2001; Jansen, Osland y Hanf, 1998), la ética ambiental debería asignar valores intrínsecos a los entes de la naturaleza y evitar la instauración de valores instrumentales que justifiquen su uso insostenible? 
Se trata desde luego de dos posiciones, una deontológica y otra utilitarista, que puede muy bien ejemplificarse con una situación que retrató Kant en "los Deberes con los Animales y el Espíritu" (Brennan \& Lo, 2008): Kant sugiere que la crueldad hacia los perros debe ser reprobada porque las personas pueden desarrollar un carácter cruel hacia los humanos. O sea, la crueldad es sancionada porque puede afectar la convivencia de los hombres, y no porque les debamos respeto a los animales mismos. Esto es un caso típico de valor instrumental de los entes naturales, que ante el evento de ser reprochado en favor de los valores intrínsecos, bien puede conducir a sin salidas cuando se trate de establecer reglas morales que, por ejemplo, prohíban el consumo de carne de res en razón del maltrato a que son expuestos aquellos animales. Pero, en el otro extremo se puede esbozar otro escenario. Si los valores instrumentales campean por las legislaciones nacionales y códigos éticos de los ciudadanos, validando cualquier intervención en la naturaleza so pretexto de contribuir al bienestar de los ciudadanos, entonces no habrá posibilidad alguna de constreñir aquellas prácticas que podrían ocasionar daños irreversibles en el medio ambiente.

En fin, se está ante dos problemas éticos ambientales fundamentales: una moral que asigna valores de respeto o valores sagrados a los entes naturales, en la cual se inscribe la mayoría de los movimientos ecológicos radicales como "ecología profunda"; y otra moral que asigna valores condicionados a los seres naturales, según la utilidad que le pueda prestar a los seres humanos, en la cual se inscribe los movimientos ecológicos reformistas como el "desarrollo sostenible". En este sentido, las preguntas que la filosofía debe ayudar a resolver son: ¿Qué perspectiva moral nos ayudaría a enfrentar el cambio climático? ¿En sociedades pluralistas, existe la posibilidad de formular una moral pragmática que incorpore tanto valores instrumentales como intrínsecos de la naturaleza, e impida que los ciudadanos crucen esa borrosa línea de la contaminación irreversible? ¿Una "ecología de la acción", esto es, un difuso ejercicio moral de ajustes progresivos entre las intenciones y los resultados ante la complejidad cotidiana de los desafíos ambientales, tal como lo propone Morin, abriría los caminos de una ética popular ambiental? (Morin, 2008)

\section{LA FILOSOFÍA DEL DERECHO}

Para la filosofía del derecho, los desafíos no dejan de ser inocuos, especialmente en lo referente al derecho intergeneracional. Siendo un asunto inicialmente de justicia distributiva que implica a las generaciones futuras, en tanto que las dinámicas sociales y económicas de hoy afectan los intereses y bienestar de aquellas (sobre todo las deudas sociales que sólo pueden ser compensadas por los ciudadanos del mañana) (Meyer, 2008), también debe ser un asunto de justicia ambiental, en tanto que las acciones contaminantes de las generaciones de hoy determinarán la 
viabilidad de las generaciones futuras. De este modo, el nudo crítico es el siguiente: ¿cómo incorporar al sistema de justicia actual los intereses y expectativas de ciudadanos que no existen, pero que deberían ser considerados en las fórmulas reguladoras del uso de los recursos naturales, en tanto que serían los más afectados por el agotamiento y devastación de nuestro patrimonio ambiental? Es más, ¿cómo establecer una defensa jurídica que asegure el futuro de los recursos naturales si el sistema de justicia apenas les asigna un valor instrumental? ¿Quién habla por y representa los intereses de... la naturaleza animada e inanimada? ¿El derecho ambiental debería instaurar una suerte de defensa animista o espiritualista de los entes naturales? Efectivamente, el debate sigue abierto, y se hace patente, especialmente, cuando se trata de valorar bienes ambientales en disputas judiciales, o cuando los gobiernos dirimen conflictos ambientales derivados de la implantación de proyectos productivos mediante la aplicación del criterio costo-beneficio, que a la sazón no podrá restituir los beneficios sacrificados de las generaciones futuras, ni tampoco valorar las especies que se extinguirán.

\section{LA FILOSOFÍA POLÍTICA}

En lo que toca a la filosofía política subsisten desafíos aún mayores. Así pues, en las distintas arquitecturas de una "sociedad bien ordenada" tanto de Habermas como de Rawls (arquitecturas que sólo abordaremos mediante su popular debate sobre el Liberalismo Político), la dimensión ambiental no es un problema estructural, y quizás se considere parte de las perspectivas morales que participan en el debate democrático, o como un componente de las distintas concepciones del bien. Siendo así las cosas, debería preguntarse si la "posición original" de Rawls no debe primero incluir las representaciones de teorías comprehensivas que antes que nada acordaran, en calidad de principio fundacional de las sociedades democráticas avanzadas, si los ciudadanos prefieren vivir en la tierra o en otro lugar. O en otras palabras, ¿no debería existir una posición original ontológica previa a la posición original que funda los valores básicos de la sociedad, en la cual los representantes debatieran en el papel de ciudadanos sin un mundo ambientalmente sostenible?

No se trata de que en la posición original se incluya el punto de vista ambiental, sino de reconocer que, la condición de posibilidad de cualquier sociedad bien ordenada es, un previo acuerdo fundacional de la sostenibilidad ambiental del planeta (para una revisión de las posiciones véanse Habermas, 1998:41-71 \& Rawls, 1998:76-143). Adicionalmente, los mecanismos de construcción de la sociedad bien ordenada, en su versión "procedimentalista" de cuño rawlsiano, o en la perspectiva de abrir espacios de deliberación pública, a decir de Habermas, no contemplan de ningún modo el debate sobre las teorías de la elección social que, a pesar nuestro, están estableciendo límites e inconsistencias de los procesos tradicionales de elección democrática (Arrow y Raynaud, 
1986) y de toma de decisiones públicas, gracias en buena parte a las investigaciones contemporáneas sobre el diseño y evaluación de políticas públicas ambientales (Munda y Nardo, 2003).

Ciertamente, las limitaciones que ofrecen los mecanismos de elección de mayorías simples o de mayorías con "peso importancia" (conocido también como participación cuantitativa) son, en resumen, las siguientes: la elección elimina casi todos los puntos de vista, o mejor, descarta las aspiraciones de N-1 candidatos; propicia escenarios de exclusión de los puntos de vista de los $\mathrm{N}$ 1candidatos; limita el debate democrático sobre las $\mathrm{N}$ propuestas $\mathrm{u}$ opciones ofrecidas (o sea, desestimula la participación cualitativa); en consecuencia con lo anterior, desarrolla una legitimación vacía del liderazgo, y crea realidades virtuales o artificiales sobre las preferencias de los candidatos (para una revisión de las consecuencias filosóficas véase Martínez, 2006).

En este sentido, Condorcet (1785) y, posteriormente, Arrow (entre los más destacados), incursionaron en métodos más consistentes con las preferencias grupales o sociales, y propusieron un mecanismo de elección según el mayor número de preferencias del electorado, conocido también como Matriz de Condorcet Corregida u Outranking Matrix. Mediante este mecanismo, el electorado puede ordenar las preferencias de los candidatos, sin eliminar así ninguna propuesta y propiciando el debate de las mismas, y determinar el candidato más preferido mediante un simple conteo de preferencias $a P b-a$ preferido $b$ - (para una ilustración práctica sobre el tema véanse algunas aplicaciones en Munda, 2004). De este modo, la propuesta ganadora será aquella que obtenga el mayor consenso posible entre el electorado.

Evidentemente, realizando pruebas podemos demostrar que el candidato que más votos recibe mediante el mecanismo de mayoría simple casi siempre es el candidato que menor consenso genera entre los electores. En suma, nuestras sociedades han empleado el peor mecanismo posible de elección para construir consensos.

\section{LA FILOSOFÍA DE LA NATURALEZA}

Finalmente, en lo que toca a la filosofía de la naturaleza, existen más disensos y enfoques que en las anteriores filosofías prácticas, dado el carácter ideológico o metafísico que han tomado en las últimas tres décadas. Así pues, podemos resumir en dos posturas el debate ambiental sobre los fundamentos últimos o fundamentos históricos de los entes de la naturaleza: en primer lugar, las posturas que infunden una visión panteísta, o animista, o monista de la naturaleza, en cuyas filas milita la "ecología profunda", la ética de la Tierra, el nuevo animismo y el realismo dialéctico (una tendencia actual del materialismo dialéctico que se funde con una especie de espiritualismo cósmico, Mantatov, 2003), y constituyen en su conjunto una metafísica ambientalista de la naturaleza; y en segundo lugar, las posturas que defienden una constitución político- 
ideológica de la naturaleza, entre las cuales se encuentran la ecocrítica y el ecofeminismo, conformando de este modo un historicismo ambientalista de la naturaleza.

En el caso de la metafísica ambientalista, a la naturaleza le es propia una armonía o equilibrio entre sus variadas fuerzas, que sólo el hombre puede interrumpir y, en algunos casos, restaurar por sus propios medios. Dado el valor intrínseco de sus entes, el hombre debe también establecer una relación simétrica y respetuosa con ella, que implique, en el escenario menos exigente, el aprovechamiento de sus frutos en consonancia con el ciclo biogeoquímico de la tierra. Romper dicho ciclo es quebrar la armonía de las fuerzas naturales. No obstante, tal simetría cosmológica es completamente disfuncional cuando se trata de introducir otras perspectivas culturales cuyos usos instrumentales de la tierra son irreductibles, esto es, proyectos productivos eficientistas y estilos de vida hedonistas. De hecho, tal filosofía de la naturaleza, cuyas "auto-evidencias culturales" son respetables, no dejan de ser la expresión de una minoría en el contexto de otras visiones de mundo mayoritarias. Su estatus de verdad y legitimidad, verificable el primero por la consistencia interna de sus enunciados y no por la evidencia empírica que, por el contrario, ha demostrado que no existe equilibrio en los ecosistemas (Sarkar, 2005), no ofrece el material suficiente para interpelar los canales de legitimación de una sociedad.

En el caso del historicismo ambiental, la naturaleza es víctima de una ideología cosificadora y machista, que tiene sus raíces en la racionalidad instrumental o en la sociedad patriarcal. La naturaleza misma ha sido desencantada y maltratada por la modernidad, lo que a su vez implica que ésta en sí misma es fuente de libertad y creatividad si el hombre se pone en relación estética o igualitarista con ella. Se trata en fin de un "dejar fluir" de las cosas naturales, muy propio de la doctrina budista, pero en una versión secularizada y romántica. En consecuencia, si la naturaleza es aquello que fluye por sí mismo evidenciando la libertad y cooperación de las cosas naturales, entonces el hombre en calidad contemplativa podrá redescubrirse a sí mismo como lo que es verdaderamente, es decir, libre y solidario. No obstante el optimismo, no es claro cómo el reencantamiento de la naturaleza o la vuelta estética a la naturaleza misma, puede refundar las premisas políticas modernas del crecimiento económico, ni cómo puede resolver los desafíos de la superpoblación humana.

\section{BORRADORES SOBRE LA FILOSOFÍA DE LA SOSTENIBILIDAD}

Existen, por supuesto, avances en los últimos veinte años sobre la ciencia de la sostenibilidad (Kates et. al., 2000), impulsados poderosamente por los procesos de toma de decisiones públicas en materia ambiental con participación ciudadana, actores políticos y conocimiento experto (Kasemir et. al. ,2002), pero con efectos 
prácticos casi inexistentes. Asimismo, se han elaborado propuestas epistemológicas que han contribuido a la fundamentación de dicha ciencia y reconocido la complejidad de los objetos de estudio (sobre esta propuesta conocida como ciencia post-normal véanse Funtowicz \& Ravetz, 1990; Funtowicz et al., 1990; Funtowicz \& Ravetz, 1994; Funtowicz et. al., 1999; Funtowicz et. al., 2002) que, evidentemente, deben integrar saberes biofísicos con dinámicas sociales, económicas, culturales y políticas. A pesar de los avances, es claro que una sistematización de sus presupuestos y consecuencias está aún por elaborarse, lo que en efecto abre una vía expedita para las investigaciones filosóficas, y exige desde ya una mínima estructura de filosofía de la sostenibilidad que sirva de soporte a la ciencia de la sostenibilidad.

En este sentido, se mencionará de modo general una propuesta de diez principios básicos para esta filosofía en proceso de construcción.

Primero: todos los objetos de investigación son complejos, esto es, no existen objetos aislados en el sistema mundo, por lo cual se debe presumir que todos están íntimamente interconectados; y si dichos objetos siguen patrones, se debe a una condición fortuita de orden a escala humana, derivada de interacciones aleatorias.

Segundo: el orden encontrado en los objetos de investigación no puede ser un orden en sí mismo, pues ello implicaría un lugar vedado para las interacciones aleatorias, o negaría el supuesto de la complejidad. El orden de dichos objetos es un orden a la medida y escala de los hombres, o mejor, son construcciones sociales y construcciones científicas. En consecuencia, el orden verificado por la ciencia y la vida cotidiana es una función de operatividad (lenguajes, normas jurídicas y costumbres) y predictividad (leyes físicas, patrones sociales e intuiciones prácticas cotidianas) de la racionalidad: operatividad que garantiza la supervivencia en el mundo cultural y natural, y predictividad que asegura la continuidad de la vida cultural y la eficiencia de toda la tecnología.

Tercero: la verdad es sólo un enunciado verificable por su operatividad y predictividad.

Cuarto: no existen funciones racionales de operatividad y predictividad que expliquen todos los órdenes posibles, es decir, no existe completitud en el conocimiento (Morin, 1998).

Quinto: los objetos sociales y naturales pueden seguir patrones lineales y no lineales.

Sexto: la operatividad y predictividad del conocimiento científico y aplicado es más eficiente si y sólo si empleamos instrumentos de investigación-acción participativa e integrada; lo que es aplicable no sólo al sistema de las ciencias humanas, sino también al sistema de las ciencias naturales. 
Séptimo: las funciones de operatividad y predictividad no pueden compensar con acciones de bienestar individual o social ningún daño ambiental que sea irreversible y que afecte a un número razonable de generaciones futuras.

Octavo: un "daño ambiental irreversible", un "número razonable degeneraciones futuras", un "umbral de población humana" y un "mínimo vital para todos" serán definidos mediante acuerdos refundadores del contrato social.

Noveno: las regulaciones de las matrices de producción y distribución de bienes y servicios (o sea, la economía) deben garantizar la implementación efectiva de los acuerdos fundacionales del principio Octavo.

Décimo: los estilos de vida contemporáneos que en concepto de todas las doctrinas morales del mundo sean contrarios a los principios Octavo y Noveno deberán ser objeto de un política de educación ambiental supra-generacional que sea lo más incluyente y respetuosa posible de sus convicciones.

\section{CONCLUSIONES}

La filosofía ambiental se debate aún en el clásico problema popperiano de demarcación entre la metafísica (panteísmo) y las ciencias positivas (validez empírica) de sus enunciados. A pesar del debilitamiento de estas últimas, en virtud de los desarrollos de la teoría de la complejidad y la ciencia post-normal, el instrumental de validación científica de las investigaciones ambientales no ha logrado superar el sistema analítico de las ciencias positivas. De hecho, los intentos de sistemas sintéticos de cuño ambiental han experimentado serias dificultades para tender puentes de comunicación entre las disciplinas socio-céntricas y bio-céntricas.

Las teleologías políticas de la filosofía ambiental, a pesar de su enorme servicio a las disciplinas militantes de cuño ambiental, han perdido de vista la necesidad de fundamentar una epistemología capaz de integrar las ciencias sociales y naturales. Ciertamente, el anterior intento de filosofía de la sostenibilidad pretende servir de gozne metodológico entre aquellos dos reinos de la ciencia. Los avances de Bertalanffy, Capra, Briggs, Peat, Maturana y Morin, apenas se pueden circunscribir a una estrategia metodológica de formulación de una suerte de analogia entis entre el mundo social y natural, con profundas raíces (a veces ignorada por ellos) en la cosmología tradicional y el materialismo dialéctico. De hecho, Bertalanffy (2007:28-29) representa todavía la mejor aproximación de integración de las ciencias con elementos de validez fundados en los hechos. 
El animismo, y especialmente, las pretensiones de substanciación de valores intrínsecos en animales con sistemas nerviosos complejos, se enfrenta aún al clásico problema wittgensteniano de los límites del lenguaje, o de la psicología de la percepción que impone límites ciertos a la "reproducción" sensorial del mundo sensible y a la "reproducción" inteligible del mundo de la apercepción (la memoria, particularmente). O sea, la imposibilidad, en el orden del lenguaje humano, de comunicarnos con los animales, suspende la posibilidad misma de reconocer "objetiva y universalmente" sus valores intrínsecos; y establecer una jerarquía de complejidades nerviosas entre los animales, como el sufrimiento, nos impone otra forma arbitraria de discriminación de los valores intrínsecos. Evidentemente, la intersubjetividad o la comunicación plena, a pesar de su imposibilidad, a decir de Nietzsche, goza al menos de una eficiencia operativa y prescriptiva que no ostenta ninguna especie no humana. Lo que en efecto no prueba concluyentemente la posibilidad de la comunicación, pero sí evidencia al menos un nivel de comunicación instrumental, tal como lo estudió a fondo Lacan.

Finalmente, aceptar el supuesto de cognición de efectos holísticos implicado en el principio de precaución (el cual sigue siendo antropocéntrico), para luego extender a todo el mundo animado e inanimado la asignación de valores intrínsecos, podría llevarnos de manera instrumental a los axiomas cognitivos del igualitarismo ecosférico o de la ecología profunda, pero no a sus axiomas deontológicos. No obstante, en este nivel, el holismo científico podría conducirnos a una mejor comprensión del mundo socionatural, pero cartesianamente escindido de un holismo moral, pues según éste, sería imposible cualquier forma de crecimiento económico y de metabolismo antropocéntrico con el mundo sensible. De hecho, sólo podríamos aspirar a la lógica ciega de la unidad dialéctica con la naturaleza, como cualquier entidad que participa sin consciencia en el equilibrio dinámico de la misma. Por ahora, indiferentemente de la perspectiva ambientalista que se esté materializando en el mundo concreto, subsiste de modo irreductible una jerarquización de valores intrínsecos asignados a los animales, una discriminación instrumental de entidades naturales, y la aceleración antrópica de los procesos de disipación de materia y energía de los ciclos biofísicos.

\section{BIBLIOGRAFÍA}

- Abram, D. (1996). "The Spell of the Sensuous", New York, Vintage Books.

- Arrow, K. J. \& Raynaud, H. (1986): "Social Choice and Multicriterion Decision Making”, Cambridge, M. I. T. Press. 
- Baker, A. M. (2007). "A Precautionary Tale: Towards a Sustainable Philosophy of Science", The Journal of Philosophy, Science \& Law, Vol. 7.

- Bertalanffy, L. V. (2007).[1968]. Teoría general de los sistemas. FCE. México.

- Brennan, A. \& Lo, Y. (2008). "Environmental Ethics”, Última consulta: mayo 2 de 2008.Recuperado de http://plato.stanford.edu/archives/spr2008/entries/ethicsenvironmentall.

- Briggs, J. \&Peat, D. (1999): "Las Siete Leyes del Caos", Barcelona, Revelaciones.

- Carpintero, O. (2006). "La Bioeconomía de GeorgescuRoegen", España, Montesinos, Ensayo.

- Carson, R. (1963). "Silent Spring", London, Hamish Hamilton.

- Collins, S. (1974). "A Different Heaven and Earth", Valley Forge, Judson Press.

- Daly, H. E. (1977). "Steady State Economics", W. H. Freeman, New York \& San Francisco.

- (1980). "Economics, Ecology, Ethics, W. H. Freeman, New York \& San Francisco.

- (1997). "Georgescu-Roegen versus Solow/Stiglitz", Ecological Economics, 22.

- Font, N. \&Subirats, J. (eds.) (2000). "Local y Sostenible. La Agenda Local en España", Barcelona, Icària.

- Funtowicz, S. O. \& Ravetz, J. R. (1990). "Uncertainty and Quality in Science for Policy", Dordrecht, Kluwer. Academic Publishers.

- (1991). "A New Scientific Methodology for Global Environmental Issues", Ecological Economics: The Science and Management of Sustainability, New York, Columbia University Press.

- (1994). "The Worth of a Songbird: Ecological Economics as a Post-Normal Science", Ecological Economics, pp. 10.

- Funtowicz, S. O.; Martínez-Alier, J.; Munda, G., \&Ravetz, J. (1999). "Information Tools for Environmental Policy under Conditions of Complexity", European Environmental Agency, Experts' Corner, Environmental Issues Series, 9.15 .

- (2002). "Multicriteria-Based Environmental Policy, H. Abaza \& A. Baranzini (eds.), Implementing Sustainable Development, Cheltenham, UNEP, Edward Elgar.

- Funtowicz, S. O.; Munda, G. \& Paruccini, M. (1990). "The Aggregation of Environmental Data Using Multicriteria Methods", Environmetrics, 1, 4.

- Gause, G. F. (1934). "The Struggle for Existence", Baltimore, Williams \& Wilkins.

- Georgescu-Roegen (1971). "The Entropy Law and the Economic Process", Harvard University Press. 

(1972). "Energy and Economic Myths", Energy and Economic Myths.

(1977). "Inequality, Limits and Growth from a Bioeconomic View Point", Review of Social Economy, 35, 3.

- Habermas, J. \&Rawls, J. (1998). "Debate sobre el Liberalismo Político", Barcelona, Paidós I. C.E./UAB, Pensamiento Contemporáneo.

- Jäeger, C.; Schüle, R.; Kasemir, B. (1999): "Focus Groups in Integrated Assessment: A Micro-cosmos for Reflexive Modernization", Innovation.12, 2.

- Jansen, A.; Osland, O. \&Hanf, K. (1998). "Environmental Challenges and Institutional Changes, An Interpretation of the Development of Environmental Policy in Western Europe", En Kenneth Hanf\& Alf-Inge Jansen, (eds.), Governance and Environment in Western Europe, Politics, Policy and Administration, Pearson Books.

- Kasemir, B.; Jäger, J.; Jäeger, C.; Gardner, M. T. (eds.) (2002). "Public Participation in Sustainability Science", Cambridge University Press.

- Kates, R. W.; Clark, W.; Corell, R.; et al, (2000). "Sustainability Science: Research and Assessment Systems for Sustainability Program", Discussion Paper, 33, Kennedy School of Government, Harvard University.

- Lenschow, A. (2001). "Environmental Policy Integration, Greening Sectoral Policies in Europe", James \& James.

- Leopold, A. (1949). "A Sand County Almanac", Oxford, Oxford University Press.

- Luke, T. W. (1997). "Ecocritique: Contesting the Politics of Nature, Economy and Culture", Minneapolis, University of Minnesota Press.

- Mantatov, V. V. (2003). "Philosophy of Sustainable Development". Última consulta: mayo 5 de 2008. Recuperado de: http://wcp2003.org/humanrights/Mantatov(Artc1).

- Martínez, A. (2006). "El PDA: Entre el Buen Gobierno y el Nuevo Gobierno Alternativo" Última consulta: 21 de mayo de 2012. Recuperado desde: Link

- Martínez, J. \& Roca, J. (2001). "Economía Ecológica y Política Ambiental", México, Fondo de Cultura Económica.

- Mathews, F. (2003). "For Love of Matter", Albany, State University of New York Press.

- Meadows D. H.; Meadows, D. L.; Randers, J. \& Behrens, W. W. (1972). "The Limits to Growth", New York, New American Library.

- Meyer, L. (2008). "Intergenerational Justice". Última consulta: $\quad$ abril $\quad 30 \quad$ de 2008. Recuperado desde: http://plato.stanford.edu/archives/spr2008/entries/justiceintergenerationall.

- Morin, E. (1998). "Introducción al Pensamiento Complejo", Barcelona, Gedisa Editorial. 
- Mumford L. (1944). "The Condition of Man", New York, Harcourt, Brace, Jovanovich.

- (1961). "The City in History", New York, Harcourt, Brace, Jovanovich.

- Munda, G. (1995). "Multicriteria Evaluation in a Fuzzy Environment", Series, Contributions to Economics, Heidelberg, Physica-Verlag.

- (2004). "Measuring Sustainability: A MultiCriterion Framework", Environment, Development and Sustainability, Kluwer AcademicPublishers.

- Munda, G. \& Nardo, M. (2003). "Mathematical Modelling of Composite Indicators for Ranking Countries", ISPRA, JRC.

- Munda, G., Nijkamp, P., \& Rietveld, P. (1995)."Qualitative Multicriteria Methods for Fuzzy Evaluation Problems", European Journal ofOperational Research, 82.

- Næss, A. (1973). "The Shallow and the Deep, Long-Range Ecology Movement", Inquiry, 16.

- Odum, H. T. (1971). "Environment, Power and Society", Wiley, New York.

- Pratley, J. E. (1989). "Towards a Philosophy of Sustainable Agriculture". Última consulta: 2 mayo de 2008. Recuperado desde: http://regional.org.au/au/roc/198991.htm.

- Renn O., Webler, T., Wiedemann, P. (eds.) (1995). "Fairness and Competence in Citizen Participation", Dordrecht, Kluwer Academic Publishers.

- Rolston, H. (1975). "Is There an Ecological Ethic?"Ethics, 85.

- Routley, R. (1973). "Is There a Need for a New, an Environmental Ethic?"Vol. 1, Sophia, Sophia Press.

- Sarkar, S. (2005). "Ecology". Última consulta: 2 mayo de $2008 . \quad$ Recuperado desde: http://plato.stanford.edu/archives/spr2008/entries/ecology/

- Shaffer, M. L. (1978). "Determining Minimum Viable Population Sizes: A Case Study of the Grizzly Bear", Disertación para obtener Ph. D., Duke University.

- Solow, R. (1997). "Reply", Ecological Economics, 22.

- Stiglitz, J. (1997). "Reply", Ecological Economics, 22.

- Stone C. D. (1972). "Should Trees Have Standing?" Southern California Law Review, 45.

- Tàbara, J. D. (2003). "Participación Cualitativa y Evaluación Integrada del Medio Ambiente", Doc. Anàl.Geogr., 42. (1998). "Citizen Participation and Equity in Global Environmental Change, the IA-FocusGroup Process", Lifestyles, Participation and Environment Workshop, Comisión Europea, Bruselas.

- Tansley, A. G. (1935). "The Use and Abuse of Vegetational Concepts and Terms", Ecology, 16.

- Vogel, S. (1996). "Against Nature: The Concept of Nature in Critical Theory, Albany, State University of New York Press. 
- Volterra, V. (1927). "Variations and Fluctuations in the Numbers of Coexisting Animal Species", In Scudo, F.M. \& Ziegler, J.R. (eds.)

- White, L. (1967). "The Historical Roots of Our Ecological Crisis", Science, 55.

1. Profesor de la Universidad del Tolima, alexandermartinezrivillas@gmail.com 